\title{
REVIEW PAPER ON MOVING OBJECT DETECTION USING ANGLE CHANGE METHOD
}

\author{
Neha Garg ${ }^{1} \&$ Er. Preeti ${ }^{2}$
}

Abstract- The detection and identify moving objects in a video sequence is very necessary concept. The currently available techniques for motion estimation can be broadly categorized into main classes: block matching methods and optical flow methods. But here detection of moving objects is detected by using Angle Change Method. Here we convert the image in to pixels and then calculate the values when the pixel change its position according to $\mathrm{x}$ axis and $\mathrm{y}$ axis. There is no problem of fixed and movable background. This paper investigates the various motion estimation algorithms utilized for video processing applications. Among the available motion estimation methods, the Lucas kanade Algorithm using changed angle change method has been utilized in this paper for detection of moving objects in a video sequence. The algorithm produces the result of Horizontal and Vertical components velocity, $u$ and $v$ respectively. This velocity is measured in the form of vectors and has been utilized to segment the moving objects from the video sequence. The algorithm has been applied to various sets of synthetic and real video sequence.

Keywords- Motion detection, Angle Change Method, optical flow, background subtraction.

\section{INTRODUCTION}

Detection and tracking moving [9] objects has many applications in the field of machine vision such as: video compression, monitoring systems, industrial control and gesture-based computer interaction.

Tracking and detecting motion of objects in a video sequence is one of the most necessary tasks of the emerging computer vision technologies. Video surveillance has become a very popular mode of monitoring, especially in secure or restricted access areas [2]. Sensors which detect the moving objects are utilized in almost all video surveillance applications. By using the estimated motion, objects can be segmented. Segmentation of moving object scan be beneficial to segregate regions of interest from the background [4].There are many ways to track the moving object. Most of them use the frame differences to analysis the moving object and obtain object boundary, which may be quite time consuming. Conventional approaches to moving objects detection include frame difference (or temporal difference) algorithms, background subtraction algorithms, optical flow algorithms and statistical learning algorithms. Various image processing techniques can be utilized to detect and track moving objects in a video sequence. These techniques can be classified into two broad classes: feature based and motion based.

- Feature based techniques differentiate moving objects from the stationary ones on the basis of one or more object features such as shape, color, profile etc [5].

- The motion based techniques (Optical Flow technique) usually compare relative or absolute motion between moving and stationary objects.

Even though these two methods remain most popular, another type of technique which has gained a lot of interest in recent times is based on artificial intelligence [7].

\section{CLASSIFICATION}

Various image processing techniques can be utilized to detect and track moving objects in a video sequence. These techniques can be categorized into two broad classes: feature based and motion based.

- Feature based techniques variousiate moving objects from the stationary ones on the basis of one or more object features such as shape, colour, profile etc.

- The motion based techniques (Optical Flow technique) usually compare relative or absolute motion between moving and stationary objects.

Even though these two methods remain most popular, another type of technique which has gained a lot of interest in recent times is based on artificial intelligence.

\footnotetext{
${ }^{1}$ Student of Master of Technology, Department of Computer Science and Engineering, Gurukul Vidyapeeth Institute of Engineering and Technology, Punjab Technical University, Punjab, India.

${ }^{2}$ Assistant Professor, Department of Computer Science and Engineering, Gurukul Vidyapeeth Institute of Engineering and Technology, Punjab Technical University, Punjab, India.
} 


\subsection{Block Matching Method-}

Block matching methods are one of the most popular feature based methods utilized to estimate motion. The method is based on the measurement of absolute motion between objects in two video frames [6]. All block matching methods start with the division of any video frame into macro-blocks of desired dimensions. The method usually uses two adjacent MPEG video frames, referred to as reference frame and target frame. The two frames are then divided into macro-blocks. These corresponding macro-blocks between the two frames are compared to estimate motion in the form of vectors. The disadvantage of this method is that it does not work when the background are identified as moved regions [8].

\subsection{Optical Flow Method-}

Optical flow is defined as an apparent motion of image brightness patterns in an image sequence. The Optical Flow Algorithm is based on relative motion rather than absolute motion, as in the case of motion vector search method. Using this method, the direction and speed of moving objects from one image to another is obtained in terms of velocity vectors (horizontal and vertical velocity vector components). Optical flow method, based on gray-scale change trend of every point on image, it can be seen as the instantaneous velocity of the point, is also an approximate estimate of the real stadium [3]. Disadvantage of this method is that this method is quite complicated and requiring a higher hardware, not suitable for real-time processing

\subsection{Space Vector Difference Method:-}

The method utilizes the space vector difference to obtain a difference map between current video frame and background model. Then, by analyzing characteristic of the difference map histogram, an adaptive threshold is automatically calculated. This method uses the concept of RGB colour technique [1]. In this red, green and blue colour are divided according to x, y and $\mathrm{z}$ axis for detection of objects. This method is applied to colour images and gives the good results but disadvantage of this method is 1 that it cannot detect the objects from background if the colour of the background and the object is same. To remove the disadvantage of this method we have a new method called Angle Change Method.

\subsection{The Principle of Space Vector Difference-}

The RGB colour space is defined chromaticities of the red, green, and blue and can produce any chromaticity that is the by those primary colours. For every pixel of a value is defined, which represents the vector space. The vector length and direction are the attributes for a vector, Thus the difference between two pixels at same images. For every position, of a current image frame, the relation exist image RGB vector associated with this position. The background RGB vector, B(r, g, b), can with the vector difference of them $\mathrm{D}(\mathrm{r}, \mathrm{g}, \mathrm{b})$ will play very necessary role in our method by the three additive primaries [1].

\section{PURPOSED METHOD}

In video surveillance we take the pictures of scenes using a camera. The original picture is divided in to pixels. Then we check the motion of a pixel according to $\mathrm{x}$ axis and $\mathrm{y}$ axis with respect to time $\mathrm{T}$. According to these axis we calculate the velocity of a object. The velocity of an object is taken as no of objects moving in a distance per unit time in an area. We can call this vector method .In this vector method we find the magnitude of vector according to $\mathrm{x}$ axis and $\mathrm{y}$ axis.

Velocity=distance/time

$\mathrm{V}=\left(\mathrm{V}^{2} \mathrm{x}^{+} \mathrm{V}^{2}\right)^{1 / 2}$

Consider a pixel of a moving object at point $\mathrm{A}$ (pixel coordinates $(\mathrm{x}, \mathrm{y})$ at time $\mathrm{t}$ ) with image brightness equal to

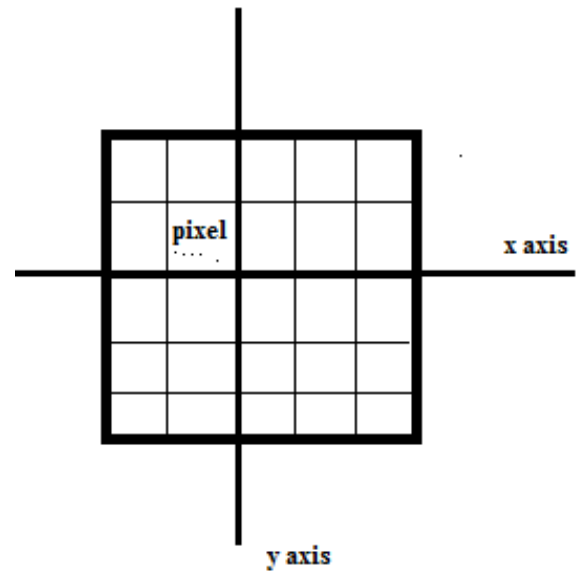

Figure1. Original picture divided into pixels and movement of pixels according to $\mathrm{x}$ axis and $\mathrm{y}$ axis.

$I(x, y, t)$. Because of object movement between frames, this pixel moves to point $B$ (new pixel coordinates being $(x+d x, y+d y)$ at time $t+d t)$; implying that the pixel coordinates $(x, y)$ change in time $d t$ to $(x+d x, y+d y)$. It is assumed that the image intensity or brightness $\mathrm{I}(\mathrm{x}, \mathrm{y}, \mathrm{t})$ of the pixel with coordinates $(\mathrm{x}, \mathrm{y})$ is constant (brightness constancy assumption) in both these frames. 
The brightness constancy assumption thus results in the following equation:

$\mathrm{I}(\mathrm{x}, \mathrm{y}, \mathrm{t})=\mathrm{I}(\mathrm{x}+\mathrm{dx}, \mathrm{y}+\mathrm{dy}, \mathrm{t}+\mathrm{dt})$

Using Taylor series expansion (only consider first order terms) the right hand side of the above results.

$\mathrm{I}(\mathrm{x}+\mathrm{dx}, \mathrm{y}+\mathrm{dy}, \mathrm{t}+\mathrm{dt})=\mathrm{I}(\mathrm{x}, \mathrm{y}, \mathrm{z})+\partial \mathrm{I} / \partial \mathrm{x} * \mathrm{dx}+\partial \mathrm{I} / \partial \mathrm{y} * \mathrm{dy}+\partial \mathrm{I} / \partial \mathrm{t} * \mathrm{dt}+\ldots$

$\partial \mathrm{I} / \partial \mathrm{x} * \mathrm{dx}+\partial \mathrm{I} / \partial \mathrm{y} * \mathrm{dy}+\partial \mathrm{I} / \partial \mathrm{t} * \mathrm{dt}=0$

Divide this $3^{\text {rd }}$ equation with $\mathrm{dt}$ and results are given below

$\partial \mathrm{I} / \partial \mathrm{x} * \mathrm{dx} / \mathrm{dt}+\partial \mathrm{I} / \partial \mathrm{y} * \mathrm{dy} / \mathrm{dt}+\partial \mathrm{I} / \partial \mathrm{t}=0$

The components according to $\mathrm{x}$ axis any $\mathrm{y}$ axis are denoted as

$\mathrm{dx} / \mathrm{dt}=\mathrm{u}$.

$\mathrm{dy} / \mathrm{dt}=\mathrm{v}$

Then the values of $u$ and $v$ are taken in equation $\quad 4$

$\partial \mathrm{I} / \partial \mathrm{x} * \mathrm{u}+\partial \mathrm{I} / \partial \mathrm{y} * \mathrm{v}=-\partial \mathrm{I} / \partial \mathrm{t}$

According to lucas kanade method we take is equation as

$\partial \mathrm{I} / \partial \mathrm{x}=\mathrm{I}_{\mathrm{x}}$

$\partial \mathrm{I} / \partial \mathrm{y}=\mathrm{I}_{\mathrm{y}}$

We put these values in equation 7

$\mathrm{I}_{\mathrm{x}} * \mathrm{u}+\mathrm{I}_{\mathrm{y}} * \mathrm{v}=-\mathrm{I}_{\mathrm{t}}$

Then we take the Threshold values. We set this values from 1 to 15 . We compare the pixel intensity with Threshold values.

This gives the best result between 7 and 9 . Object is clearly identified between these values.

\section{PROBLEM DEFINITION/OBJECTIVES}

To detect the moving objects in a video surveillance, Detection of moving objects is very necessary task. For this many methods are utilized like background subtraction method, optical flow method, Space vector difference method, subtraction of adjacent frames method. Background subtraction method compares the current frame with the background model that is constantly updated, it is inconsistent with the background are identified as moving region. This is the limitation of this method. Optical flow method based on grey scale change trend of every point of an image. It can be seen as instantaneous velocity of the points. But this method is very complicated and needs more hardware. Subtraction of adjacent frames method is utilized to detect the position and shape of moving objects by calculating the difference between two adjacent frames. This method is simple but more sensitive to background noise. Space vector difference method is utilized to detect the moving objects in a colour space. This method utilizes the space vector difference to obtain a difference map between current video frame and background model. So histogram and Threshold is automatically calculated. The Threshold values detect the moving objects and also reduce the noise. But if the colour of the background and the object is same then detection of objects is not possible. For this we select the method of changing Angle. For removing the noise we utilized Gaussian pyramid which utilized the magnitude of $\mathrm{x}$ and $\mathrm{y}$ axis

\section{CONCLUSION AND FUTURE WORK}

In this paper a new approach Angle Change Method is implemented. This algorithm is implemented for removing the disadvantage of space vector difference method in colouring images where the background and the current object have same colour. There is no need to take the background model because it only depends on the current pixel's movement according to axis. In this Lucas kanade algorithm is utilized for calculating the first order derivative of a pixel because we can't take only extreme position but also the values between the first and the last end. In future our focus is on camera quality and sound. If the threshold of an object increases suddenly then it generates the effect on sound when the alarm is generated at detection of object.

\section{REFERENCES}

[1] Quian, Zhen. and Huang, Debao. "Moving objects detection based on Space Vector Difference”, IEEE, International conference of Mechatronics and Automation, vol.646-651 (2011).

[2] Zheng, Xiaoshi. and Zhao, Yanling. "An automatic moving object detection algorithm for video surveillanceapplications", IEEE, International conference on Embedded software and systems, vol. 541-543 (2009).

[3] Shafie, A. A. and Hafiz, Fadhlan. "Moving object detection using optical flow", World Academy of science and technology, vol. 559-561 (2009).

[4] Liu, Feng. and Gleicher, Micchael. "Learning colour and locality cues for moving object detection and segmentation" (2009).

[5] Elhabian, Shireen.Y. and Ahmed, Sumaya.H. "Moving object detection in spatial domain using background removal techniques", Recent patents on computer science, vol. 1. (2009).

[6] Lu, Nan. and Wang, Jihong."An improved motion detection method for real time surveillance”, International journal of computer science (2008).

[7] Bugeau, Aurelie. and Perez, Patrick. "Detection and Segmentation of moving objects in highly dynamic scenes", France conference of computer and science (2007).

[8] Davis, James. W. and Sharma,Vinay. "Background-Subtraction using contour-based fusion of thermal and visible imagery", Computer vision and image understanding, vol. 162-182 (2007).

[9] A. Yilmaz, O. Javed and M. Shah “Object tracking: a survey”, ACM Computing Surveys, vol. 38, no. 4, pp. 1-45, 2006. 